\title{
NUMERICAL AND THEORETICAL ANALYSIS OF AN AWARENESS COVID-19 EPIDEMIC MODEL VIA GENERALIZED ATANGANA-BALEANU FRACTIONAL DERIVATIVE
}

\author{
Isa Abdullahi Babal ${ }^{1}$, Idris Ahmed ${ }^{2}$, Qasem M. Al-Mdallal ${ }^{3}$, Fahd Jarad ${ }^{4}$, Salisu Yunusa ${ }^{5}$ \\ ${ }^{1}$ Department of Mathematical Science, Bayero University Kano \\ Kano, Nigeria \\ ${ }^{2}$ Department of Mathematics, Sule Lamido University, Kafin Hausa \\ Jigawa State, Nigeria \\ ${ }^{3}$ Department of Mathematical Sciences, United Arab Emirates University \\ Al Ain, Abu Dhabi, UAE \\ ${ }^{4}$ Department of Mathematics, Çankaya University \\ Ankara, 06790 Turkey \\ ${ }^{5}$ Department of Counsellor Education and Counselling Psychology, Universiti Putra Malaysia \\ Serdang, Malaysia \\ isababa7@yahoo.com,idrisahamedgml1988@gmail.com,q.almdallal@uaeu.ac.ae \\ fahd@cankaya.edu.tr, sykafinhausa@gmail.com
}

Received: 17 August 2021; Accepted: 11 November 2021

\begin{abstract}
In this paper, a COVID-19 Awareness model in the setting of a generalized fractional Atangana-Baleanu derivative is proposed. The existence and uniqueness of a solution of the proposed fractional-order model are investigated under the techniques of fixed point theorems. In addition, we perform the predictor-corrector method to find its numeric solutions and present the graphs of the various solutions using different values of the parameters embodied in the derivative.
\end{abstract}

MSC 2010: 26A33, 34A12, 34A43, 34D20

Keywords: existence and uniqueness, generalized $A B C$ fractional derivative, fixed point theorems, numerical simulations

\section{Introduction}

Coronavirus (COVID-19) was first detected in a city called Wuhan in China in December 2019, and it then spread exponentially in almost every part of the globe. The virus is known as severe acute coronavirus syndrome 2 (SARSCoV-2) [1]. COVID-19 has no prompt diagnosis and vaccine, according to the World Health Organization (WHO, 2020) and evolves into a global pandemic that causes substantial morbidity and death. 1,603,428 confirmed cases and 356,440 recoveries from the disease were recorded as of 9 April 2020 with 95,714 deaths worldwide [2]. 
As of June 10, 2020, there are 13,464 laboratory-confirmed cases of COVID-19 with 364 deaths, recovered and discharged 4,206 while 8,893 ware undergoing treatment, in Nigeria [3]. COVID-19 is a new disease with the most destructive consequences in the world, and its existence and transmission create uncertainty, fear, and anxiety among the general population. Anxiety is the breeding place for hate and discrimination the victims [4]. Since the recurrence of the pandemic, social stigma has occurred among individuals. It is paramount that we resist this stigma because it might cause people to hide and avoid reporting themselves if they have the virus which would contribute to the spread of the virus. During COVID-19, WHO is offering advice and solutions to public esquires to help people to overcome anxiety, discrimination, and stigma. The 1st reported case of coronavirus in Nigeria was confirmed on February $27^{\text {th }}$ by an Italian man who arrived from Milan to Nigeria, he stayed in the country for almost two days before being isolated. He'd traveled through Lagos and visited other places in the world [5].

Roy et al. [4] shows that only $29.5 \%$ of the participant are aware of the multiple modes in which the virus could spread, such as touching, kissing, sneezing, and through food in which only $43 \%$ of respondents considered COVID-19 to be highly contagious. In the line with this, the public must be aware to minimize the spread of the virus. It was also shown that during this pandemic, more than eighty percent of the candidates felt the need for medical assistance from mental health professionals and doctors to resolve psychological and other emotional issues. In line with the above, there is a need for an awareness campaign to combat and control the pandemic within the populace by using print and non-print media as suggested by a counseling psychologist.

On the flip side, fractional calculus is to be one of the most interesting branches of mathematics which have prepossessed the interest of many researchers because of the results vested after these researchers used fractional operators to model reallife phenomena [6-8]. All the fractional operators had singularities in their kernels till 2015. This was aforethought to be a handicap for various applications. Due to Caputo and Fabrizio [9], that year witnessed the birth of a new fractional derivative having a nonsingular kernel. This was followed by the birth of a mathematically strong Atangana-Baleanu fractional derivative [10] which contains a type of Mittag-Leffler function having one parameter in its kernel. A generalized Atangana-Baleanu was then proposed in [11] with a 3-parameter Mittag-Leffler function in its kernel. Applications of the latter derivative can be seen in [12].

Thanks to the efficiency of this operator in modeling aspects and due to the applications of certain fractional operators in epidemic mathematical models mentioned above, this paper examines the awareness model suggested by Baba and Baleanu [13] in the sense of a system of nonlinear differential equations through the generalized 
$\mathrm{ABC}$ fractional derivative described by

$$
\begin{aligned}
{ }^{A B C} \mathscr{D}_{t_{0}}^{\sigma, v, \rho} S(t) & =\Lambda-\beta S I-\lambda S A-\bar{d} S+\psi I+\Theta S_{A}, \\
{ }^{A B C} \mathscr{D}_{t_{0}}^{\sigma, v, \rho} I(t) & =\beta S I-(\psi+\alpha+\bar{d}) I, \\
{ }^{A B C} \mathscr{D}_{t_{0}}^{\sigma, v, \rho} S_{A}(t) & =\lambda S A-\bar{d} S_{A}-\Theta S_{A}, \\
{ }^{A B C} \mathscr{D}_{t_{0}}^{\sigma, v, \rho} A(t) & =\mu I-\phi A .
\end{aligned}
$$

equipped with the appropriate initial conditions

$$
S\left(t_{0}\right) \geq 0, I\left(t_{0}\right) \geq 0, S_{A}\left(t_{0}\right) \geq 0, A\left(t_{0}\right) \geq 0
$$

where ${ }^{A B C} \mathscr{D}_{t_{0}}^{\sigma, v, \rho}(\cdot)$ is the generalized ABC-fractional operator of order $(0<\sigma \leq 1)$. The model takes into account the following clue. The population $N(t)$ is generally split into three main classes. The common Susceptible class as $S(t)$, the Infective class as $I(t)$ and the Susceptible class that have prior knowledge of the disease as $S_{A}(t)$. Finally, we define the cumulative density of the awareness program as $A(t)$. It is also believed that an increase in the number of Infectives brings about an increase in the density of the awareness program. The parameters are well explained in the Table 1.

Table 1. Parameters description

\begin{tabular}{|c|c|}
\hline Parameter & Description \\
\hline$\Lambda$ & Rate of recruitment of new members into susceptible population \\
$\beta$ & Infectious contact rate \\
$\lambda$ & Rate of awareness dissemination \\
$\Theta$ & Rate of recovery \\
$\alpha$ & Infectious death rate \\
$\bar{d}$ & Natural death rate \\
$\psi$ & Rate at which aware individuals are transferred to susceptible class \\
$\mu$ & Rate of implementing the awareness program \\
$\phi$ & Depletion rate due to social problems and ineffectiveness of the program \\
\hline
\end{tabular}

\section{Preliminaries}

For the simplicity of the notation we take

$$
\chi=\left(S, I, S_{A}, A\right) .
$$

and $\mathbb{E}=C\left(\left[t_{0}, b\right], \mathbb{R}\right)$ denotes the Banach space of all continuous functions from $\left[t_{0}, b\right]$ into $\mathbb{R}$ with the norm defined by

$$
\|\chi\|_{\mathbb{E}}=\sup _{t \in\left[t_{0}, b\right]}|\chi(t)|,
$$


where $|\chi(t)|=|S(t)|+|I(t)|+\left|S_{A}(t)\right|+|A(t)|$ and $\chi:\left[t_{0}, b\right] \times \mathbb{R}^{4} \rightarrow \mathbb{R}$ is a given continuous function.

Definition 1 [14] Let $0<\sigma \leq 1, v>0$ and $\rho>0$. The operator

${ }^{A B} \mathscr{J}_{t_{0}}^{\sigma, v, \rho} g(t)=\sum_{k=0}^{\infty}\left(\begin{array}{c}\rho \\ k\end{array}\right) \frac{\sigma^{k}}{N(\sigma)(1-\sigma)^{k-1}} \frac{1}{\Gamma(\sigma i+1-v)} \int_{t_{0}}^{t}(t-s)^{\sigma i-v} g(s) d s, t>t_{0}$,

is called the generalized $\mathrm{AB}$ fractional integral of order $\sigma$ of a function $g$ where $N(\cdot)>0$ denotes the normalizing function such that $N(0)=N(1)=1$.

Definition $2[11,14]$ Let $0<\sigma \leq 1, v>0$ and $\rho \in \mathbb{R}$. The generalized ABC fractional derivative of order $\sigma$ with the lower limit $t_{0}$ for a function $g$ is defined by

$$
{ }^{A B C} \mathscr{D}_{t_{0}}^{\sigma, v, \rho} g(t)=\frac{N(\sigma)}{1-\sigma} \int_{t_{0}}^{t} E_{\sigma, v}^{\rho}(p, t-s) g^{\prime}(s) d s, t>t_{0},
$$

provided the function $g$ differentiable on $\left[t_{0},+\infty\right), E_{\sigma, v}^{\rho}(p, t-s)$ is a two parameter Mittag-Leffler function defined by

$$
E_{\sigma, v}^{\rho}(p, t-s)=\sum_{k=0}^{\infty} p^{k} \frac{(t-s)^{\sigma k+v-1}(\rho)_{k}}{\Gamma(\sigma k+v) k !},
$$

with $p=\frac{-\sigma}{1-\sigma},(\rho)_{k}=\rho(\rho+1) \cdots(\rho+k-1)$.

Lemma 1 [14] If $\sigma \in(0,1), v>0, \rho \in \mathbb{C}$ and $p=\frac{-\sigma}{1-\sigma}$. Then

$$
{ }^{A B} \mathscr{J}_{t_{0}}^{\sigma, v, \rho A B C} \mathscr{D}_{t_{0}}^{\sigma, v \rho} g(t)=g(t)-g\left(t_{0}\right) .
$$

Thus, the given problem

$$
\left\{\begin{array}{l}
\left(\begin{array}{l}
\left.{ }^{A B C} \mathscr{D}_{0^{+}}^{\sigma, v, \rho} g\right)(t)=h(t), \\
g\left(t_{0}\right)=g\left(t_{0}\right),
\end{array}\right.
\end{array}\right.
$$

possesses the given solution of the form:

$$
g(t)=g\left(t_{0}\right)+\sum_{k=0}^{\infty}\left(\begin{array}{l}
\rho \\
k
\end{array}\right) \frac{\sigma^{k}}{N(\sigma)(1-\sigma)^{k-1}} \frac{1}{\Gamma(\sigma i+1-v)} \int_{t_{0}}^{t}(t-s)^{\sigma i-v} h(s) d s .
$$

\section{Existence and uniqueness of solutions}

The existence and uniqueness of nonlinear fractional differential equations play a key role in the study of the qualitative analysis of fractional differential equations, see [15]. Utilizing the concepts of Schaefer's and Banach's fixed point theorems, 
we present here in this section the existence and uniqueness of the solutions of the fractional model (1). Rewrite systems (1) in the form:

$$
\begin{aligned}
{ }^{A B C} \mathscr{D}_{t_{0}}^{\sigma, v, \rho} S(t) & =\mathscr{A}_{1}\left(t, S, I, S_{A}, A\right), \\
{ }^{A B C} \mathscr{D}_{t_{0}}^{\sigma, v, \rho} I(t) & =\mathscr{A}_{2}\left(t, S, I, S_{A}, A\right), \\
{ }^{A B C} \mathscr{D}_{t_{0}}^{\sigma, v, \rho} S_{A}(t) & =\mathscr{A}_{3}\left(t, S, I, S_{A}, A\right), \\
{ }^{A B C} \mathscr{D}_{t_{0}}^{\sigma, v, \rho} A(t) & =\mathscr{A}_{4}\left(t, S, I, S_{A}, A\right),
\end{aligned}
$$

where

$$
\begin{aligned}
& \mathscr{A}_{1}\left(t, S, I, S_{A}, A\right)=\Lambda-\beta S I-\lambda S A-\bar{d} S+\psi I+\Theta S_{A}, \\
& \mathscr{A}_{2}\left(t, S, I, S_{A}, A\right)=\beta S I-(\psi+\alpha+\bar{d}) I, \\
& \mathscr{A}_{3}\left(t, S, I, S_{A}, A\right)=\lambda S A-\bar{d} S_{A}-\Theta S_{A}, \\
& \mathscr{A}_{4}\left(t, S, I, S_{A}, A\right)=\mu I-\phi A .
\end{aligned}
$$

Thus, the proposed fractional model (1) takes the form:

$$
\left\{\begin{array}{l}
{ }^{A B C} \mathscr{D}_{t_{0}}^{\sigma, v, \rho} \Theta(t)=\mathscr{R}(t, \Theta(t)) ; \quad t \in\left[t_{0}, b\right], 0<\sigma<1, \\
\Theta\left(t_{0}\right)=\Theta_{0} \geq 0
\end{array}\right.
$$

on condition that

$$
\left\{\begin{array}{l}
\Theta(t)=\left(S, I, S_{A}, A\right)^{T} \\
\Theta(0)=\left(S_{0}, I_{0}, S_{A_{0}}, A_{0}\right)^{T} \\
\mathscr{R}(t, \Theta(t))=\left(\Theta_{i}\left(t, S_{0}, I_{0}, S_{A_{0}}, A_{0}\right)\right)^{T}, \quad i=1, \cdots, 4
\end{array}\right.
$$

where $(\cdot)^{T}$ represents the transpose operation. From Lemma 1, problem (11) is given by

$$
\begin{aligned}
\Theta(t) & =\Theta_{0}+{ }^{A B} \mathscr{I}_{t_{0}}^{\sigma, v, \rho} \mathscr{R}(t, \Theta(t)) \\
& =\Theta_{0}+\sum_{k=0}^{\infty}\left(\begin{array}{l}
\rho \\
k
\end{array}\right) \frac{\sigma^{k}}{N(\sigma)(1-\sigma)^{k-1}} \frac{1}{\Gamma(\sigma i+1-v)} \int_{t_{0}}^{t}(t-s)^{\sigma i-v} \mathscr{R}(s, \Theta(s)) d s
\end{aligned}
$$

Consider the fractional operator $T: \mathbb{E} \rightarrow \mathbb{E}$ defined by

$$
(T \Theta)(t)=\Theta_{0}+\sum_{k=0}^{\infty}\left(\begin{array}{l}
\rho \\
k
\end{array}\right) \frac{\sigma^{k}}{N(\sigma)(1-\sigma)^{k-1}} \frac{1}{\Gamma(\sigma k+1-v)} \int_{t_{0}}^{t}(t-s)^{\sigma k-v} \mathscr{R}(s, \Theta(s)) d s .
$$


Obviously, the operator $T$ is well defined and the fixed points of the operator $T$ are the solution of the fractional model (1). For simplicity, we denote

$$
\Delta=\left\{\begin{array}{l}
\sum_{k=0}^{\infty}\left(\begin{array}{l}
\rho \\
k
\end{array}\right) \frac{\sigma^{k}}{N(\sigma)(1-\sigma)^{k-1}} \frac{1}{\Gamma(\sigma k+2-v)}\left(b-t_{0}\right)^{\sigma k+1-v}, \quad v \neq 1, \\
\sum_{k=0}^{\infty}\left(\begin{array}{l}
\rho \\
k
\end{array}\right) \frac{\sigma^{k}}{N(\sigma)(1-\sigma)^{k-1}} \frac{1}{\Gamma(\sigma k+1)}\left(b-t_{0}\right)^{\sigma k}, \quad v=1 .
\end{array}\right.
$$

Now, we state and prove the existence of solution of the proposed fractional model (1) by employing the concept of Schaefer's fixed point theorem.

Theorem 1 Suppose

$\left(H_{1}\right)$ There exist $\eta_{1}, \eta_{2} \in \mathbb{E}$ with $\eta_{1}^{*}=\sup _{t \in[0, b]} \eta_{1}(t), \eta_{2}^{*}=\sup _{t \in[0, b]} \eta_{2}(t)<1$ such that

$$
|\mathscr{R}(t, \Theta)| \leq \eta_{1}(t)+\eta_{2}(t)|\Theta(t)|, \text { for } t \in\left[t_{0}, b\right], \Theta \in \mathbb{E} .
$$

Then there exists at least one solution of the proposed model (1).

PROOF The proof is based on the following steps:

Step 1: The operator $T$ is continuous.

Let $\Theta_{n}$ be a sequence such that $\Theta_{n} \rightarrow \Theta$ in $\mathbb{E}$. Then for each $t \in\left[t_{0}, b\right]$

$$
\begin{aligned}
\left|\left(T \Theta_{n}\right)(t)-(T \Theta)(t)\right| \leq & \sum_{k=0}^{\infty}\left(\begin{array}{l}
\rho \\
k
\end{array}\right) \frac{\sigma^{k}}{N(\sigma)(1-\sigma)^{k-1}} \frac{1}{\Gamma(\sigma k+1-v)} \\
& \times \int_{t_{0}}^{t}(t-s)^{\sigma k-v}\left|\mathscr{R}\left(s, \Theta_{n}(s)\right)-\mathscr{R}(s, \Theta(s))\right| d s \\
\leq & \Delta\left\|\mathscr{R}\left(\cdot, \Theta_{n}(\cdot)\right)-\mathscr{R}(\cdot, \Theta(\cdot))\right\|_{\mathbb{E}} .
\end{aligned}
$$

Since $\mathscr{R} \in C([0, b], \mathbb{R})$, then $\left\|\left(T \Theta_{n}\right)-(T \Theta)\right\|_{\mathbb{E}} \longrightarrow 0$, as $n \rightarrow+\infty$.

Step 2: $T$ maps bounded set into a bounded set.

To see this, we show that for $r>0$, there exists a $\mathscr{L}>0$ constant such that $\Theta \in \mathscr{B}_{r}=$ $\left\{\Theta \in \mathbb{E}:\|\Theta\|_{\mathbb{E}} \leq r\right\}$, then $\|T \Theta\|_{\mathbb{E}} \leq \mathscr{L}$. Now,

$$
\begin{aligned}
|(T \Theta)(t)| \leq & \left|\Theta_{0}\right|+\sum_{k=0}^{\infty}\left(\begin{array}{l}
\rho \\
k
\end{array}\right) \frac{\sigma^{k}}{N(\sigma)(1-\sigma)^{k-1}} \frac{1}{\Gamma(\sigma k+1-v)} \\
& \times \int_{t_{0}}^{t}(t-s)^{\sigma k-v}|\mathscr{R}(s, \Theta(s))| d s .
\end{aligned}
$$


By $\left(H_{1}\right)$, we get

$$
\begin{aligned}
|(T \Theta)(t)| \leq & \Theta_{0}+\left(\eta_{1}^{*}+\eta_{2}^{*}\|\Theta\|\right) \sum_{k=0}^{\infty} \\
& \times\left(\begin{array}{l}
\rho \\
k
\end{array}\right) \frac{\sigma^{k}}{N(\sigma)(1-\sigma)^{k-1}} \frac{1}{\Gamma(\sigma k+1-v)} \int_{t_{0}}^{t}(t-s)^{\sigma k-v} d s \\
\leq & \mathscr{L}<+\infty .
\end{aligned}
$$

Step 3: $T$ maps bounded sets into equicontinuous set.

Assume $t_{1}, t_{2} \in\left[t_{0}, b\right]$, with $t_{1} \geq t_{2}$, and $\mathscr{B}_{r}$ be a bounded set as defined above. For any $\Theta \in \mathscr{B}_{r}$, yields

$$
\begin{aligned}
\mid(T \Theta)\left(t_{1}\right) & -(T \Theta)\left(t_{2}\right) \mid \\
\leq & \mid \sum_{k=0}^{\infty}\left(\begin{array}{l}
\rho \\
k
\end{array}\right) \frac{\sigma^{k}}{N(\sigma)(1-\sigma)^{k-1}} \frac{1}{\Gamma(\sigma k+1-v)} \\
& \times \int_{t_{0}}^{t_{1}}\left[\left(t_{1}-s\right)^{\sigma k-v}-\left(t_{2}-s\right)^{\sigma k-v}\right] \mathscr{R}(s, \Theta(s)) d s \mid \\
& +\left|\sum_{k=0}^{\infty}\left(\begin{array}{l}
\rho \\
k
\end{array}\right) \frac{\sigma^{k}}{N(\sigma)(1-\sigma)^{k-1}} \frac{1}{\Gamma(\sigma k+1-v)} \int_{t_{1}}^{t_{2}}\left(t_{2}-s\right)^{\sigma k-v} \mathscr{R}(s, \Theta(s)) d s\right| \\
& \longrightarrow 0, \text { as } t_{2} \rightarrow t_{1} .
\end{aligned}
$$

Thus, Step $1-3$ and the Arzelá Ascoli theorem, we deduce that the operator $T$ is completely continuous.

Step 4: A prior bounds.

Next, we show that the

$$
\mathscr{U}=\{\Theta \in \mathbb{E}: \Theta=v(T \Theta), 0<v<1\},
$$

is a bounded set.

Let $\Theta \in \mathscr{U}, \Theta=o(T \Theta)$ for some $0<o<1$. Now, for $t \in\left[t_{0}, b\right]$, we get

$$
\begin{aligned}
\Theta(t)= & v\left[\Theta_{0}+\sum_{k=0}^{\infty}\left(\begin{array}{l}
\rho \\
k
\end{array}\right) \frac{\sigma^{k}}{N(\sigma)(1-\sigma)^{k-1}} \frac{1}{\Gamma(\sigma k+1-v)}\right. \\
& \left.\times \int_{t_{0}}^{t}(t-s)^{\sigma k-v} \mathscr{R}(s, \Theta(s)) d s\right] .
\end{aligned}
$$

This implies that by $\left(H_{1}\right)$ and for each $t \in\left[t_{0}, b\right]$ yields

$$
|\Theta(t)| \leq|(T \Theta)(t)|<+\infty .
$$

Hence, $\mathscr{U}$ is bounded. Therefore, the proof is completed. 
Next, we state and prove the existence result based on the Banach contraction principle.

Theorem 2 Assume the function $\mathscr{R} \in C([0, b], \mathbb{R})$ and maps bounded subset of $[0, b] \times$ $\mathbb{R}^{4}$ into relatively compact subsets of $\mathbb{R}$. Besides, there exists $L_{\mathscr{R}}>0$ constant such that $\left(H_{1}\right)\left|\mathscr{R}\left(t, \Theta_{1}(t)\right)-\mathscr{R}\left(t, \Theta_{2}(t)\right)\right| \leq L_{\mathscr{R}}\left|\Theta_{1}(t)-\Theta_{2}(t)\right| ;$ for all $t \in[0, b]$ and each $\Theta_{1}, \Theta_{2} \in \mathbb{E}$. Then there exists a unique solution of (14) (consequently, model (1)) provided that $\Delta L_{\mathscr{R}}<1$.

PROOF To construct a neighborhood of radius $r$, we let $\sup _{t \in[0, b]}\|\mathscr{R}(t, 0)\|=\mathscr{E}<\infty$ and choose

$$
r \geq \frac{\Delta \mathscr{E}+\left\|\Theta_{0}\right\|}{1-\Delta \mathscr{E}}
$$

Thus, it is enough to show that $T \mathscr{B}_{r} \subset \mathscr{B}_{r}$, where $\mathscr{B}_{r}=\{\Theta \in \mathbb{E}:\|\Theta\| \leq r\}$. So, for any $\Theta \in \mathscr{B}_{r}$, we have

$$
\begin{aligned}
|(T \Theta)(t)| \leq & \Theta_{0}+\sum_{k=0}^{\infty}\left(\begin{array}{l}
\rho \\
k
\end{array}\right) \frac{\sigma^{k}}{N(\sigma)(1-\sigma)^{k-1}} \frac{1}{\Gamma(\sigma k+1-v)} \int_{t_{0}}^{t}(t-s)^{\sigma k-v}|\mathscr{R}(s, \Theta(s))| d s \\
\leq & \Theta_{0}+\sum_{k=0}^{\infty}\left(\begin{array}{l}
\rho \\
k
\end{array}\right) \frac{\sigma^{k}}{N(\sigma)(1-\sigma)^{k-1}} \frac{1}{\Gamma(\sigma k+1-v)} \\
& \times \int_{t_{0}}^{t}(t-s)^{\sigma k-v}[|\mathscr{R}(s, \Theta(s))-\mathscr{R}(s, 0)|+|\mathscr{R}(s, 0)|] d s \\
\leq & r
\end{aligned}
$$

which implies $T \mathscr{B}_{k} \subset \mathscr{B}_{k}$.

Next, we let $\Theta_{1}, \Theta_{2} \in \mathbb{E}$. Then for any $t \in\left[t_{0}, b\right]$, yields

$$
\begin{aligned}
\mid\left(T \Theta_{1}\right)(t)-\left(T \Theta_{2}\right)(t) \leq & \sum_{k=0}^{\infty}\left(\begin{array}{l}
\rho \\
k
\end{array}\right) \frac{\sigma^{k}}{N(\sigma)(1-\sigma)^{k-1}} \frac{1}{\Gamma(\sigma k+1-v)} \\
& \times \int_{t_{0}}^{t}(t-s)^{\sigma k-v}\left|\mathscr{R}\left(s, \Theta_{1}(s)\right)-\mathscr{R}\left(s, \Theta_{2}(s)\right)\right| d s \\
\leq & L_{\mathscr{R}}\left\|\Theta_{1}-\Theta_{2}\right\|_{\mathbb{E}} \sum_{k=0}^{\infty}\left(\begin{array}{l}
\rho \\
k
\end{array}\right) \frac{\sigma^{k}}{N(\sigma)(1-\sigma)^{k-1}} \frac{1}{\Gamma(\sigma k+1-v)} \\
& \times \int_{t_{0}}^{t}(t-s)^{\sigma k-v} d s \\
\leq & \Delta L_{\mathscr{R}}\left\|\Theta_{1}-\Theta_{2}\right\|_{\mathbb{E}},
\end{aligned}
$$

Therefore, the there exists a unique solution of the proposed model (1). 


\section{Equilibrium solutions of the model}

Setting equation in (1) to zero and solving the system simultaneously, we obtain two equilibrium solutions, Disease free equilibrium $E_{0}$ and Endemic equilibrium $E_{1}$, where:

$$
E_{0}=\left\{S^{0}, I^{0}, S_{A}^{0}, A^{0}\right\}=\left\{\frac{\Lambda}{d}, 0,0,0\right\}
$$

and

$$
E^{1}=\left\{S^{1}, I^{1}, S_{A}^{1}, A^{1}\right\}
$$

Where,

$$
\begin{gathered}
S^{1}=\frac{\alpha+\gamma+d}{\beta} \\
I^{1}=\frac{\phi\left[\Lambda \beta d+\Lambda \beta \theta-\left(\gamma d^{2}+\theta \gamma d+\alpha d^{2}+\theta \alpha d+\theta d^{2}+d^{3}\right)\right]}{\beta \phi \alpha d+\beta \phi \alpha \theta+\beta \phi d^{2}+\beta \phi \theta d+\lambda \gamma \mu \theta+\lambda \mu d^{2}} \\
S_{A}^{1}=\frac{\lambda \mu(\alpha+\gamma+d)\left(\Lambda \beta-d \gamma-d \alpha-d^{2}\right)}{\beta\left(\beta \phi \alpha d+\beta \phi \alpha \theta+\beta \phi d^{2}+\beta \phi \theta d+\lambda \gamma \mu \theta+\lambda \mu d^{2}\right)} \\
A^{1}=\frac{\mu\left[\Lambda \beta d+\Lambda \beta \theta-\left(\gamma d^{2}+\theta \gamma d+\alpha d^{2}+\theta \alpha d+\theta d^{2}+d^{3}\right)\right]}{\beta \phi \alpha d+\beta \phi \alpha \theta+\beta \phi d^{2}+\beta \phi \theta d+\lambda \gamma \mu \theta+\lambda \mu d^{2}}
\end{gathered}
$$

It can be seen that $I^{1}, S_{A}^{1}, A^{1}$ are biologically meaningful only if $\frac{\Lambda \beta}{d(d+\alpha+\gamma)}>1$.

Define $R_{0}$, basic reproduction ratio, to be $\frac{\Lambda \beta}{d(d+\alpha+\gamma)}$. Thus the endemic equilibrium $E_{1}$ exists only if $R_{0}>1$.

\section{Numerical interpretation and graphical analysis}

The scheme is a predictor-corrector method. The full details of the numerical scheme is presented in [12]. More precisely, the approximate solution of the initial value problem

$$
{ }_{0}^{A B C} D^{\sigma, v, \rho} x(t)=g(t, x(t)), x(0)=x_{0}, t \in(0, T],
$$


where $\alpha \in(0,1)$, is given by $x_{m} \approx x\left(t_{m}\right)$ where $t_{m}=m h(h$ is the step size and $m \in \mathbb{N})$. The approximate solution $x_{m}$ is given by

$$
\begin{gathered}
x_{m}=x_{0}+\sum_{j=0}^{m-1} c_{j}^{m} g_{j}, \quad \text { where } c_{j}^{m}=\sum_{k=0}^{\infty}\left(h^{\sigma k-v+1} A_{k}^{\sigma, v, \rho} r_{k, j}^{m}\right), \\
r_{k, j}^{m}= \begin{cases}\xi_{k}^{m}-w_{k, 1}^{m, 0}, & \text { if } j=0, \\
w_{k, j}^{m, 1}-w_{k, j+1}^{m, 0}, & \text { if } 1 \leq j \leq m-2, \\
w_{k, m-1}^{m, 1}, & \text { if } j=m-1 .\end{cases}
\end{gathered}
$$

Here, $A_{k}^{\sigma, v, \rho}, w_{k, j}^{m, i}$ and $\xi_{k}^{m}$ are, respectively, given by

$$
\begin{gathered}
A_{k}^{\sigma, v, \rho}=\left(\begin{array}{l}
\rho \\
k
\end{array}\right) \frac{\sigma^{k}}{B(\sigma)(1-\sigma)^{k-1}} \frac{1}{\Gamma(\sigma k-v+1)}, \\
w_{k, j}^{m, i}=(m-j+i) \frac{(m-j)^{\sigma k-v+1}-(m-j-1)^{\sigma k-v+1}}{\sigma k-v+1} \\
+\frac{(m-j-1)^{\sigma k-v+2}-(m-j)^{\sigma k-v+2}}{\sigma k-v+2}, \\
\xi_{k}^{m}=\frac{m^{\sigma k-v+1}-(m-1)^{\sigma k-v+1}}{\sigma k-v+1} .
\end{gathered}
$$

It should be advised that the step size used in the present calculations is $h=0.1$, whereas the following initial conditions and parameters values [13] have been as given below:

$$
\begin{aligned}
& S(0)=\frac{19999685}{a a}, I(0)=\frac{3145}{a a}, S_{A}(0)=\frac{19999685}{a a}, A(0)=\frac{200}{a a}, a a=10000, \Lambda=400, \\
& \beta=0.0000157, \lambda=0.0002, \Theta=0.16979, \alpha=0.03275, \bar{d}=0.0096, \psi=0.2, \\
& \mu=0.0005 \text { and } \phi=0.06 .
\end{aligned}
$$

Figure 1: From the results and figures described here, it can be claimed that the decrease of the fractional derivative order $\sigma$ reduces the number of each population phase (except for the Susceptible Population fraction as expected) and flattens the curves to the limit in each population phase.

Figure 2: It can be noticed that reducing the rate of adoption of the awareness program below the rate of depletion of the program contributes to the prevalence of the disease in the population. On the other hand, the rise in the rate of implementation of the awareness program above the rate of depletion of the program tends to dis- 
ease prevention. This shows the importance of implementing the awareness-raising campaign, especially in underdeveloped countries such as Nigeria.
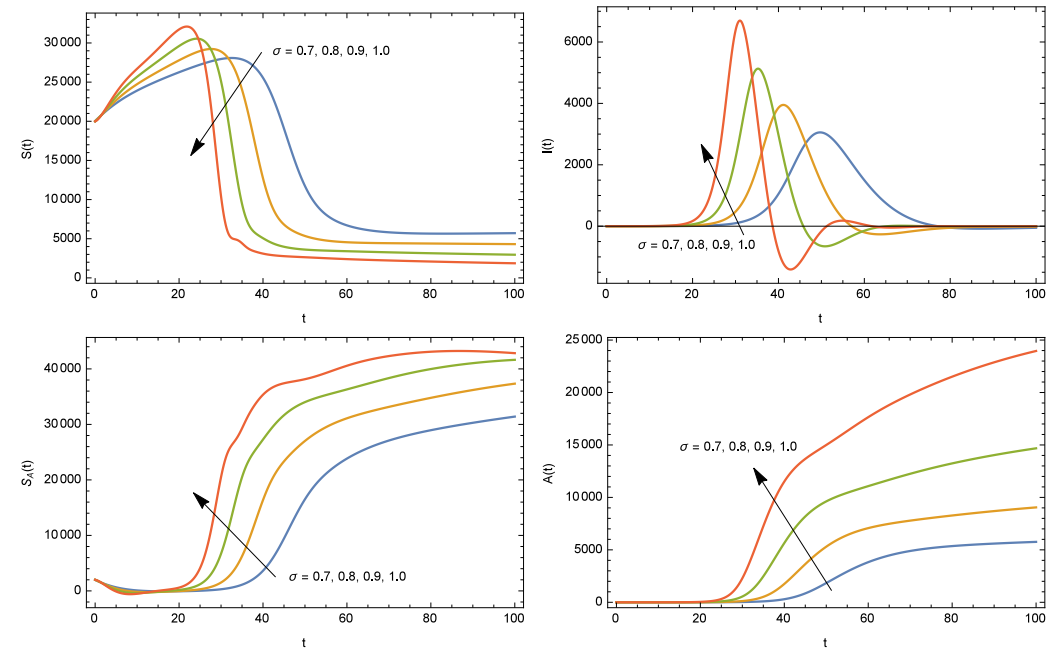

Fig. 1. Graphs of the solution trajectories at $\rho=1, v=0.5$ for different fractional order $\sigma$
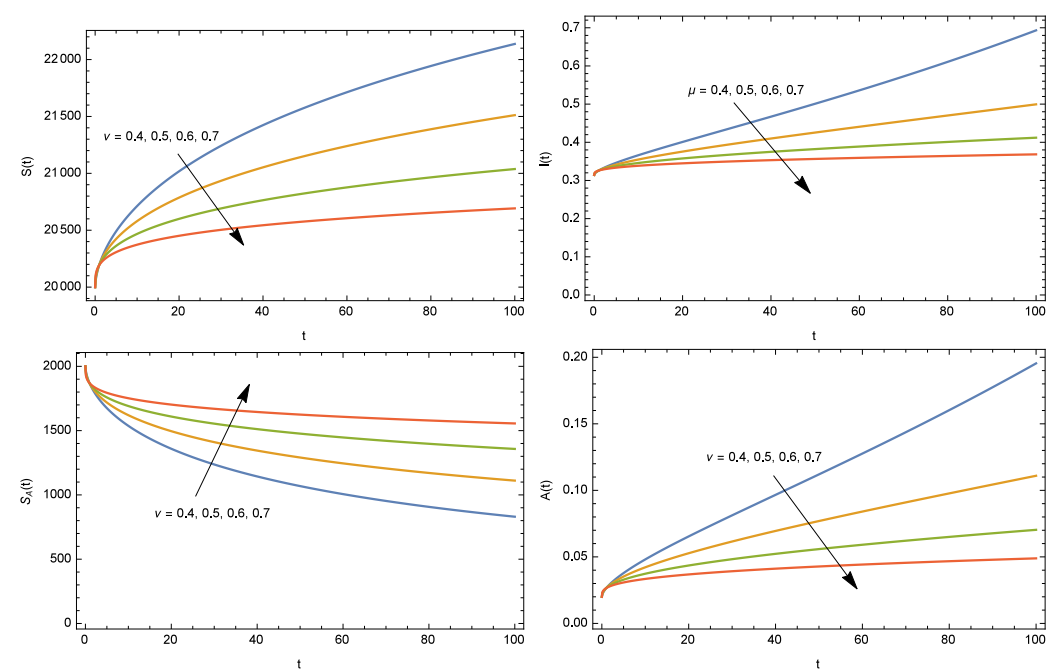

Fig. 2. Graphs of the solution trajectories at $\rho=1, \sigma=0.7$ for different values of $v$

\section{Conclusion}

In this paper, an extension of the awareness model to the fractional-order using generalized $\mathrm{ABC}$ fractional derivative has been studied. We investigate the theoretical properties of the proposed fractional-order model such as the existence and uniqueness of the solution using fixed point theorems. Besides, the numerical simulations 
are carried out via an effective numerical scheme to analyze the dynamic behavior of the fractional-order model while varying the fractional order. It has been shown from the depicted figures that the introduced fractional-order model in the sense of the generalized $\mathrm{ABC}$ operator provides more flexible results than the corresponding integer order.

\section{References}

[1] World Health Organization. Novel coronavirus diseases 2019, https://www.who.int/emergencies/ diseases/novel-coronavirus-2019, Accessed: 2020-05-05.

[2] World Health Organization. Report of the WHO-China Joint Mission on Coronavirus Disease 2019 (COVID-19), February 2020, https://www.who.int/docs/defaultsource/coronaviruse/ who-china-jointmission-on-covid-19-final-report.pdf, Accessed: 2020-05-05.

[3] Nigeria Centre for Disease Control. COVID-19 Outbreak in Nigeria: Situation Report, https://ncdc.gov.ng/diseases/sitreps/?cat=14, Accessed: 2020-05-05.

[4] Roy, D., Tripathy, S., Kar, S.K., Sharma, N., Verma, S.K., \& Kaushal, V. (2020). Study of knowledge, attitude, anxiety \& perceived mental healthcare need in Indian population during COVID-19 pandemic. Asian Journal of Psychiatry, 102083.

[5] Effiong, A.I., Nseobot, I.R., Johnny, A.E., Frank, E.I., Abere, O.J., Essien, M.O., Ukpong, E.S., $\&$ others. (2020). Assessment of Nigerian television authority (NTA) ongoing programme awareness campaigns on corona virus in Nigeria. Electronic Research Journal of Social Sciences and Humanities, 2.

[6] Podlubny, I. (1999). Fractional Differential Equations: An Introduction to Fractional Derivatives, Fractional Differential Equations, to Methods of their Solution and Some of their Applications (Vol. 198). Elsevier.

[7] Ahmed, I., Baba, I.A., Yusuf, A., Kumam, P., \& Kumam, W. (2020). Analysis of Caputo fractional-order model for COVID-19 with lockdown. Advances in Difference Equations, 2020(1), 1-14.

[8] Khan, H., Gómez-Aguilar, J.F., Alkhazzan, A., \& Khan, A. (2020). A fractional order HIV-TB coinfection model with nonsingular Mittag-Leffler Law. Mathematical Methods in the Applied Sciences, 43(6), 3786-3806.

[9] Caputo, M., \& Fabrizio, M. (2015). A new definition of fractional derivative without singular kernel. Progress in Fractional Differentiation and Applications, 1(2), 87-92.

[10] Atangana, A., \& Baleanu, D. (2016). New fractional derivatives with nonlocal and non-singular kernel: Theory and application to heat transfer model. Thermal Science, 20(2), 763-769.

[11] Abdeljawad, T., \& Baleanu, D. (2018). On fractional derivatives with generalized Mittag-Leffler kernels. Advances in Difference Equations, 2018(1), 468.

[12] Abdeljawad, T., Hajji, M.A., Al-Mdallal, Q.M., \& Jarad, F. (2020). Analysis of some generalized ABC - Fractional logistic models. Alexandria Engineering Journal, 59(4), 2141-2148.

[13] Baba, I.A., \& Baleanu, D. (2020). Awareness as the most effective measure to Mitigate the spread of COVID-19 in Nigeria. Cmc-Computers Materials \& Continua, 65(3), 1945-1957.

[14] Abdeljawad, T. (2019). Fractional operators with generalized Mittag-Leffler kernels and their iterated differintegrals. Chaos: An Interdisciplinary Journal of Nonlinear Science, 29(2), 023102.

[15] Ahmed, I., Kumam, P., Shah, K., Borisut, P., Sitthithakerngkiet, K., \& Demba, M.A. (2020). Stability results for implicit fractional pantograph differential equations via $\phi$-Hilfer fractional derivative with a nonlocal Riemann-Liouville fractional integral condition. Mathematics, 8(1), 94. 